\title{
Video-Assisted Thoracic Surgery Thymectomy: Subxiphoid Approach
}

\author{
Sukki Cho \\ Department of Thoracic and Cardiovascular Surgery, Seoul National University Bundang Hospital, Seongnam, Korea
}

\section{ARTICLE INFO}

Received June 1, 2021

Accepted July 16, 2021

Corresponding author

SukKi Cho

Tel $82-31-787-7132$

Fax 82-31-787-4050

E-mail tubincho@snu.ac.kr

ORCID

https://orcid.org/0000-0002-9309-8865

\begin{abstract}
In this paper, I present the technique of subxiphoid single-port video-assisted thoracic surgery (VATS) thymectomy for thoracic surgeons to perform this procedure safely. This procedure is indicated for all anterior mediastinal masses and may be extended to lung cancer. The patient is placed in the lithotomy position, and the operator should be on the midline. Below the xiphoid process, a skin incision is made $4-5 \mathrm{~cm}$ horizontally at a single thumb's width down. Under two-lung ventilation, $\mathrm{CO}_{2}$ is insufflated, maintaining $10 \mathrm{~mm}$ $\mathrm{Hg}$. The fat tissue and thymic tissue are all resected from the sternum and pericardium between both phrenic nerves using an articulated grasper and an energy device. After retrieval of the mass with a wrap bag, a Jackson-Pratt drain is inserted instead of a chest tube. One of the advantages of this procedure is less postoperative pain than intercostal VATS. The subxiphoid approach can be used for bilateral pneumothorax, bilateral pulmonary metastasectomy, and simple lobectomy for both upper lobes and the right middle lobe.
\end{abstract}

Keywords: Thymectomy, Subxiphoid, Video-assisted thoracic surgery, Single port

\section{Introduction}

Minimally invasive surgery for anterior mediastinal tumors includes the bilateral intercostal, cervical, and subxiphoid approaches. The intercostal approach was first performed by Landreneau et al. [1] in 1992, and is now the most popular approach. The cervical approach was initially performed by Cooper et al. [2] in 1988, but further use of this approach has not been reported in Korea. The subxiphoid approach was first performed by Kido et al. [3] in 1999 and was further advanced by Suda et al. [4]. In this paper, I would like to elaborate on single-port video-assisted thoracic surgery (VATS) thymectomy through the subxiphoid approach for anterior mediastinal tumors, with the goal of enabling beginners to perform this procedure safely.

\section{Indications}

This method is indicated for almost all anterior mediastinal masses, except masses with vascular invasion (especially invasive thymomas). Funnel chest, cardiomegaly, and a large tumor $(>5 \mathrm{~cm})$ are relative contraindications. In ad- dition to anterior mediastinal masses, this approach is also highly suitable for lymph node metastasis to the internal mammary chain or cardiophrenic angle. Some surgeons have used this technique for lung cancer surgery, but in my opinion, doing so is premature because of the great difficulty of performing both lower lobectomy and subcarinal lymph node dissection, similar to the conventional VATS procedure.

\section{Anatomy of the subxiphoid area}

The anterior mediastinum is located between the body of the sternum in the front and the anterior pericardium posteriorly (Fig. 1). It contains loose connective tissue, mediastinal fat, and the body of the thymus.

\section{Procedure}

\section{Anesthesia}

In principle, a single-lumen endotracheal tube can be used because one-lung ventilation is not necessary. Howev- 


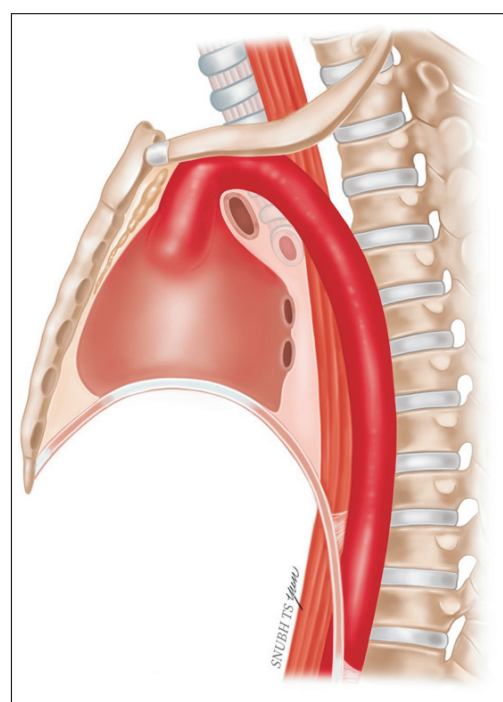

Fig. 1. Mediastinum. The anterior mediastinum is located between the body of the sternum in the front and the anterior pericardium posteriorly.

er, it is convenient to use a double-lumen endotracheal tube if the tumor is large or lung invasion is suspected. Intensive arterial monitoring is required because of the possibility of $\mathrm{CO}_{2}$ retention with $\mathrm{CO}_{2}$ insufflation and cardiac compromise during surgery or when the tumor is removed. Because the operation time is not long, it is not necessary to insert a Foley catheter or to catheterize the femoral, jugular, or subclavian vein in the absence of vascular invasion.

\section{Position}

The lithotomy position is preferable because the operator should be on the midline (Fig. 2). Both arms should be at attention because sternotomy may be required in some situations. The drape can expose the upper abdomen, sternal notch, and mid-axillary line on both sides.

\section{Surgical instruments}

(1) Wound retractor

(2) Multiport (Lapsingle version)

(3) $30^{\circ}$ thoracoscope $(5 \mathrm{~mm}$ or $10 \mathrm{~mm}$ )

(4) Grasper $(5 \mathrm{~mm})$, as long as possible $(>30 \mathrm{~cm})$ : An articulating grasper is the best, such as the Artisential grasper.

(5) Energy device: Ligasure, a Harmonic scalpel, or Thunderbeat are options, but if possible, the Maryland tip shape is preferable and it should be as long as possible $(>35 \mathrm{~cm})$.

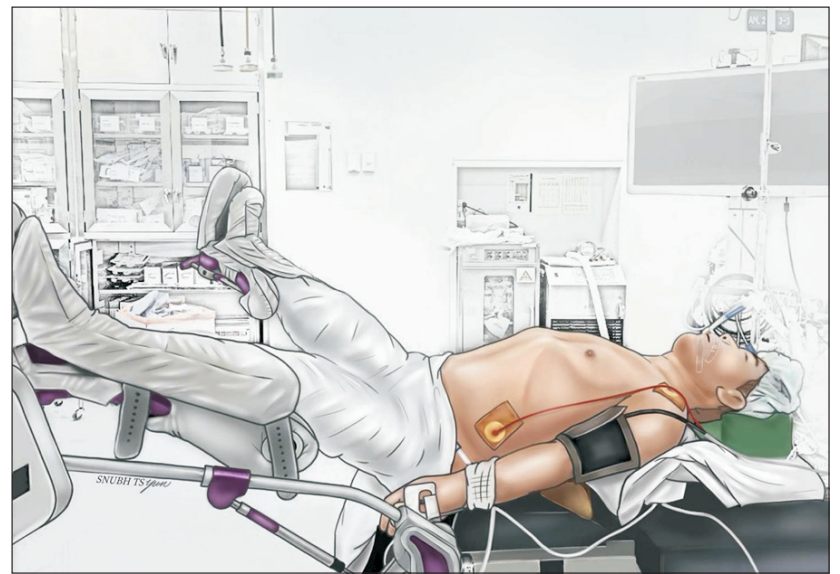

Fig. 2. Patient position. The lithotomy position is preferable, and the operator stands between the patient's legs.

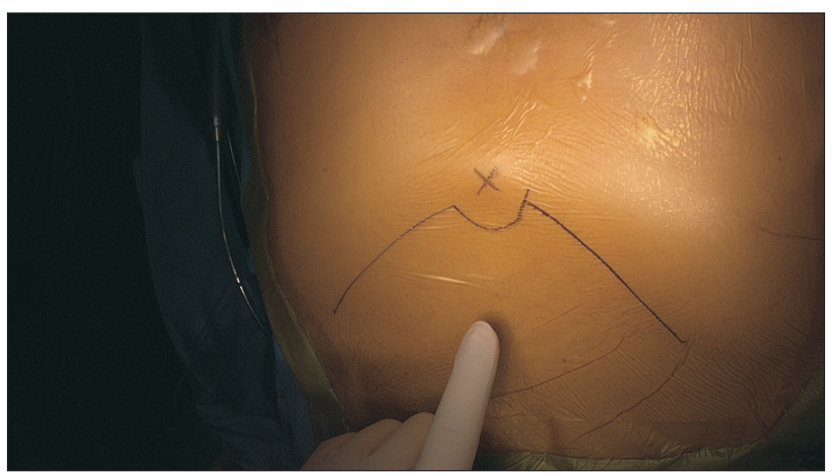

Fig. 3. Skin incision. A 5-cm horizontal incision is made below the xiphoid process.

(6) Vinyl bag, medium or large size

\section{Incision and port placement}

\section{Incision (Fig. 3)}

A skin incision is made $4-5 \mathrm{~cm}$ horizontally at a single thumb's width down from the xiphoid process. At first, it is better to be sure to make a long enough incision, and the incision size can then be gradually reduced with practice. There are 2 methods of skin incision: vertical and horizontal. The advantages of a vertical incision are that it is possible not to transect the rectus muscle, and if sternotomy is also performed, the skin incision is connected. The advantage of a horizontal incision is that it spreads slightly better on the lateral side, thus reducing collisions between instruments within a single port. Therefore, the choice between a vertical and horizontal incision should be made according to the situation. 


\section{Port placement}

After finding the xiphoid process, the rectus muscle is transected about $1 \mathrm{~cm}$ to both sides and further dissection is performed $360^{\circ}$ under the xiphoid process (Fig. 4). Using the index finger as in sternotomy, sufficient additional dissection is performed under the sternum. Adding a wound protector, the subxiphoid area is further dissected using a monopolar Bovie under thoracoscopic guidance. The Lapsingle is added and $\mathrm{CO}_{2}$ insufflation is started, maintaining $10 \mathrm{~mm} \mathrm{Hg}$ (Fig. 5). The position of the multiport and the location of the port for instruments are very important. The bilateral pleurae are opened and both lungs continue to be ventilated while maintaining the pressure of $\mathrm{CO}_{2}$ at around $10 \mathrm{~mm} \mathrm{Hg}$ (Fig. 6).

\section{Thymectomy}

When sufficient space is available for the subxiphoid ap-

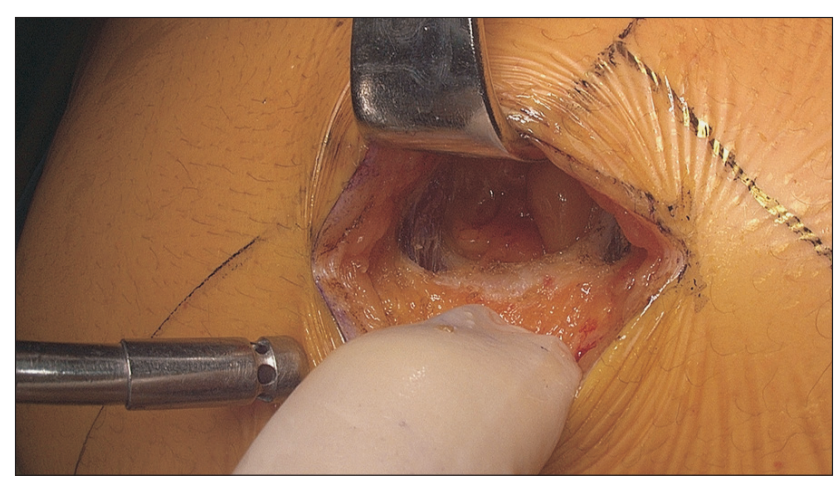

Fig. 4. Before port placement. The rectus muscle is transected about $1 \mathrm{~cm}$ horizontally and further dissection is performed $360^{\circ}$ under the xiphoid process. proach, the next step is to drop the thymic tissue from the sternum. The boundary between the 2 sides is formed by the mammary vein, and dissection proceeds upward, closely adhering to the sternum. The mediastinal pleura is opened by reaching as far as possible where the mammary vein on both sides inserts into the innominate veins. After confirming that there is no bleeding, the thymic and pericardial fat is again dissected, as well as pleural fat from the inlet of the incision. If myasthenia gravis (MG) is present, more complete dissection will be necessary. During dissection, it is necessary to check the course of both phrenic nerves (Fig. 7). In particular, if there is a suspicion that the thymic tumor invades the surrounding organs, they should

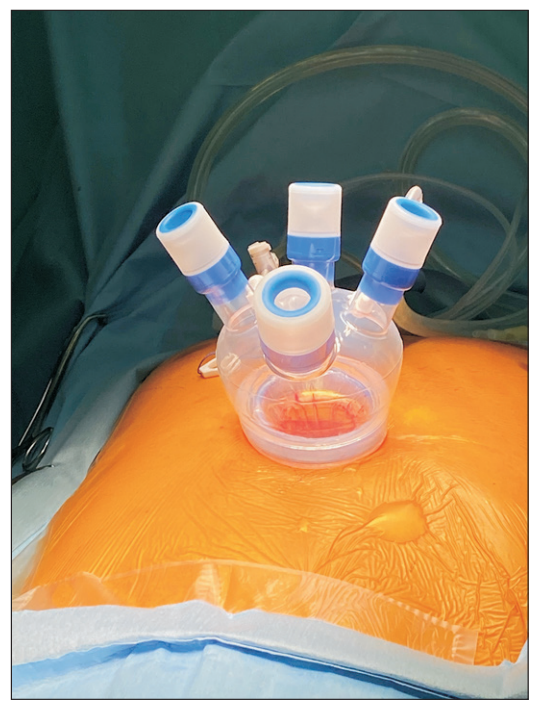

Fig. 5. After port placement. The multiport is added and $\mathrm{CO}_{2}$ insufflation is started, maintaining $10 \mathrm{~mm} \mathrm{Hg}$.
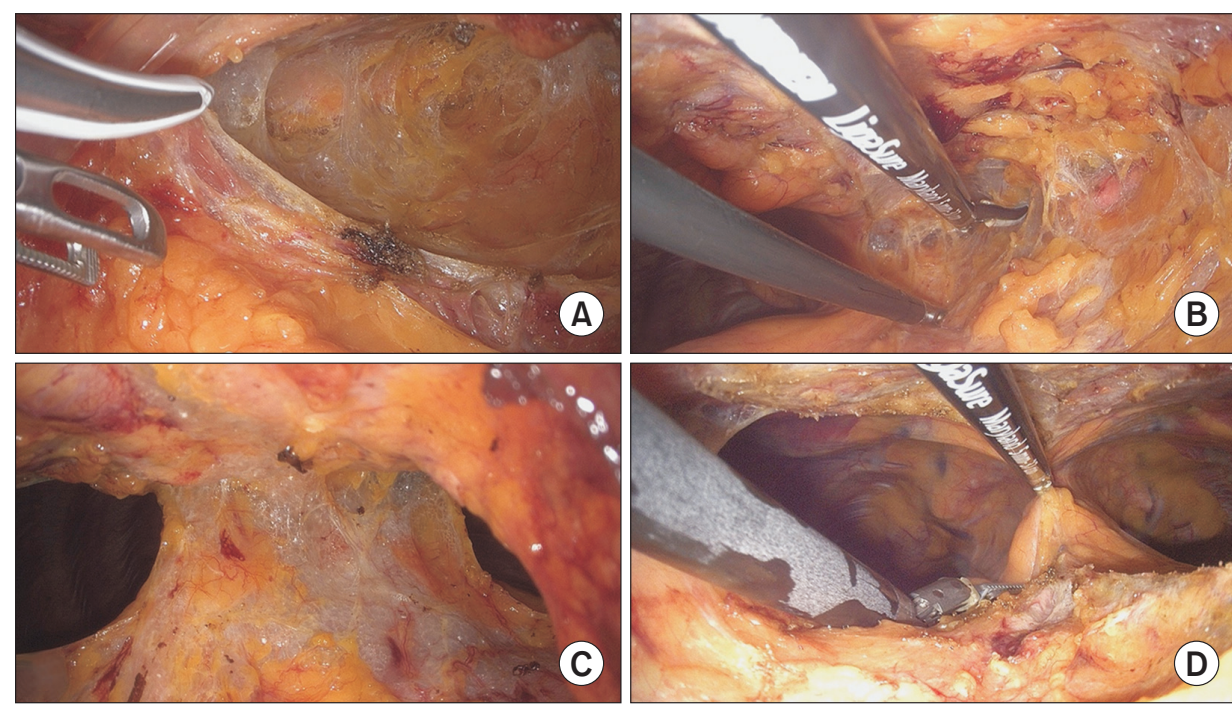

Fig. 6. Open pleura. Dissection of the substernal tissue using an energy device $(A)$, opening of the right pleura (B), opening of the left pleura $(\mathrm{C})$, and dissection of the substernal thymic tissue (D). 


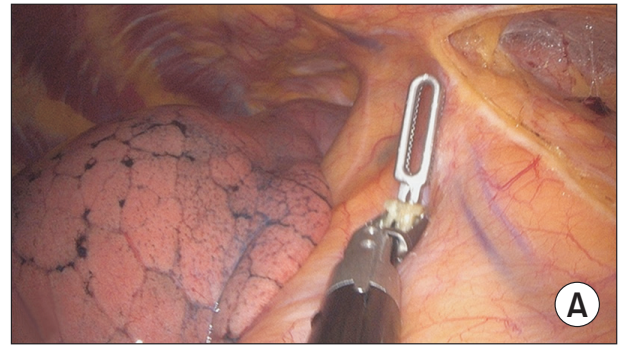

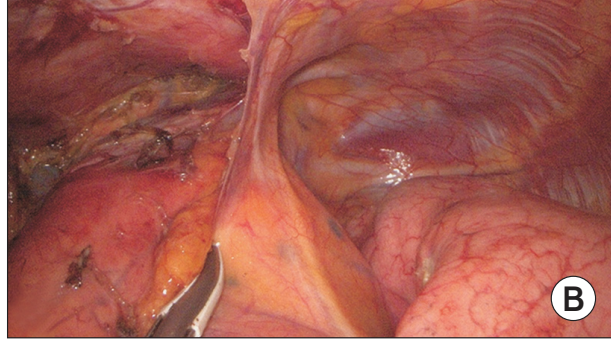

B Fig. 7. Phrenic nerve. Right phrenic nerve $(A)$ and left phrenic nerve $(B)$.
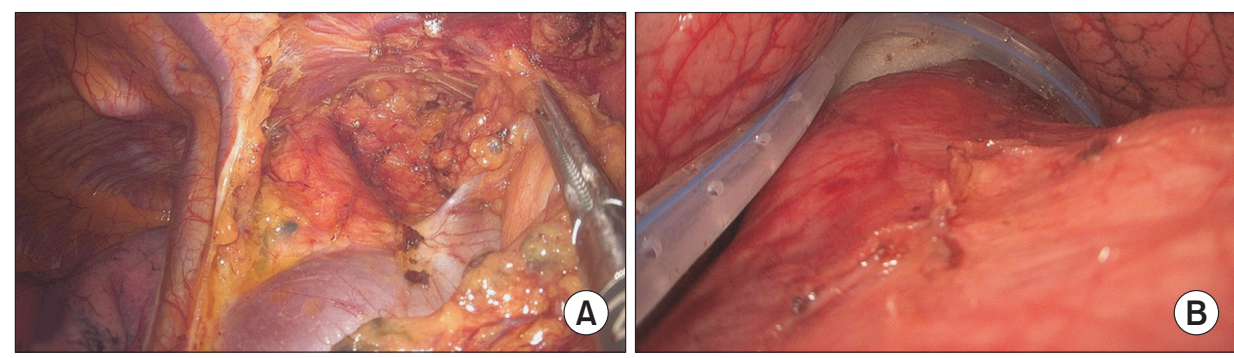

Fig. 8. Thymectomy. (A) Both upper poles are removed and (B) a Jackson-Pratt drain is positioned after retrieval of the mass.

be resected together to the greatest extent possible. Since it is quite possible for both lungs to have adhesions or invasions, an endostapler is used to resect them en bloc instead of being forced to dissect them from the mass. When encountering the innominate vein, the thymic tissue is lifted and one proceeds upward while dissecting the front of the vein. There is no branch in this area, so there is no major problem with dissecting the vessel. Next, the left upper pole is dissected. The reason for this is that if the operator is right-handed, it seems safer empirically. Furthermore, since the left pole is larger than the right, one can think of the point of removing the left pole as the farthest place during surgery. Small branches can be clipped, but this step may vary depending on the energy device used. $\mathrm{CO}_{2}$ insufflation is usually used, so it may seem that there are no areas of small bleeding, but when $\mathrm{CO}_{2}$ is cut off at the end of surgery, bleeding may occur; therefore, I recommend using a clip if possible. The left pole is removed from the top of the innominate vein, one proceeds to the right, the tissue is dissected in front of the trachea, and the right pole is removed from the front of the left innominate artery.

After total thymectomy, a wrap bag is inserted through one of the multiports, and the thymus, including the mass and adjacent fat tissue, is retrieved from the chest. It is important to note that if the tumor is large and hard, it will press upon the heart as it is removed through a narrow space; therefore, the patient's blood pressure and heart rhythm should be carefully observed.

When observing the surgical field after removing the tu- mor, there is no temporary $\mathrm{CO}_{2}$ in the thoracic cavity, so if there is a blood vessel for which proper hemostasis was not achieved, bleeding is visible. In the absence of bleeding, depending on the intraoperative findings of thymic disease, it is recommended to check for pleural metastasis under one-lung ventilation. There is no need to insert a chest tube; at this point, I insert a Jackson-Pratt (J-P) drain and close the surgical field layer by layer (Fig. 8).

\section{Postoperative management}

Immediately after the operation, a chest posteroanterior radiograph should be checked in the operating room to determine whether there is pneumothorax. If pneumothorax is present, temporary suction can be applied to the J-P drain until the air leak is stopped. On postoperative day 1 , the amount and color of the drainage are checked. If there is no evidence of chyle and the amount is less than 200 $\mathrm{mL}$, the J-P drain is removed. Special medications, including painkillers, are not necessary unless the patient has MG.

\section{Advantages}

One of the advantages of this approach is that it can reduce the frequency of bleeding caused by damage to the innominate vein, and the probability of successful hemostasis is very high without an open procedure because of the good visibility. In addition, it is very easy to perform median sternotomy without changing the patient's position 
when it is difficult to proceed with the subxiphoid approach.

In the 1-sided VATS approach, if there are pleural adhesions, almost all adhesions must be released, and the air leakage is often severe due to lung damage caused by adhesiolysis. However, the subxiphoid approach does not cause lung damage because only a small amount of adhesiolysis gives enough vision of the area to be operated on; therefore, chest tube insertion is not required.

Postoperative pain is also an inevitable complication even after 1-sided VATS. However, the subxiphoid approach causes significantly less postoperative pain than 1-sided VATS, because the intercostal nerve is not damaged by the surgical instruments during surgery, and the intercostal or other thoracic muscles are not incised.

\section{Disadvantages}

A special instrument is required to operate in a narrow space. Most of the instruments used in VATS procedures are straight, so surgery is very difficult for patients with an exceptionally depressed sternum. Thus, it can be very useful to have instruments with multiple types of articulations. Although energy devices have a straight body, there is no particular inconvenience because the tip is curved.

Suction cannot be used freely because $\mathrm{CO}_{2}$ insufflation is required. There is a risk of cardiac and pulmonary compromise depending on the pressure and flow of $\mathrm{CO}_{2}$.

\section{Conclusion}

In the near future, the subxiphoid approach can be applied to bilateral pneumothorax, bilateral pulmonary metastasectomy (especially for metastases on both upper lobes), and simple lobectomy for both upper lobes and the right middle lobe.

\section{Conflict of interest}

No potential conflict of interest relevant to this article was reported.

\section{Acknowledgments}

I would like to thank Nayun Lee for drawing the figures (Figs. 1,2) in this manuscript.

\section{ORCID}

Sukki Cho: https://orcid.org/0000-0002-9309-8865

\section{Supplementary materials}

Supplementary materials can be found via https://doi. org/10.5090/jcs.21.052. Supplementary Video 1. A 44-yearold male patient had an anterior mediastinal tumor. The tumor size was $40 \mathrm{~mm}$ and no invasion of the vessel was found on a contrast computed tomography scan. The clinical diagnosis was a thymoma without myasthenia gravis. This video shows total thoracotomy performed by subxiphoid single-port video-assisted thoracic surgery. The pathological examination showed World Health Organization type $\mathrm{AB}$ thymoma (Masaoka-Koga stage IIA). The patient was discharged a day after surgery.

\section{References}

1. Landreneau RJ, Dowling RD, Castillo WM, Ferson PF. Thoracoscopic resection of an anterior mediastinal tumor. Ann Thorac Surg 1992;54:142-4

2. Cooper JD, Al-Jilaihawa AN, Pearson FG, Humphrey JG, Humphrey HE. An improved technique to facilitate transcervical thymectomy for myasthenia gravis. Ann Thorac Surg 1988;45:242-7.

3. Kido T, Hazama K, Inoue Y, Tanaka Y, Takao T. Resection of anterior mediastinal masses through an infrasternal approach. Ann Thorac Surg 1999;67:263-5.

4. Suda T, Sugimura H, Tochii D, Kihara M, Hattori Y. Single-port thymectomy through an infrasternal approach. Ann Thorac Surg 2012;93:334-6. 